\title{
A model with two inert scalar doublets
}

\section{A.C.B. Machado*, V. Pleitez}

Instituto de Física Teórica-Universidade Estadual Paulista, R. Dr. Bento Teobaldo Ferraz 271, Barra Funda, São Paulo - SP, 01140-070, Brazil

\section{A R T I C L E I N F O}

\section{Article history:}

Received 16 June 2015

Accepted 23 October 2015

Available online 31 October 2015

\section{Keywords:}

Inert-doublets model

Dark-matter

\begin{abstract}
A B S T R A C T
We consider an extension of the standard model (SM) with three $S U(2)$ scalar doublets and discrete $S_{3} \otimes \mathbb{Z}_{2}$ symmetries. The irreducible representation of $S_{3}$ has a singlet and a doublet, and here we show that the singlet corresponds to the SM-like Higgs and the two additional $S U(2)$ doublets forming a $S_{3}$ doublet are inert. In general, in a three scalar doublet model, with or without $S_{3}$ symmetry, the diagonalization of the mass matrices implies arbitrary unitary matrices. However, we show that in our model these matrices are of the tri-bimaximal type. We also analyzed the scalar mass spectra and the conditions for the scalar potential is bounded from below at the tree level. We also discuss some phenomenological consequences of the model.
\end{abstract}

(c) 2015 Elsevier Inc. All rights reserved.

\section{Introduction}

In 2012 it was discovery at the LHC a neutral spin-0 resonance with properties (mass and couplings) that are compatible, within the experimental error, with those of the scalar SM-Higgs boson [1,2]. However, on the one hand, there is no experimental evidence confirming that only one of such sort of scalars does exist. On the other hand, there are experimental evidence, e.g. the existence of Dark Matter (DM) and neutrinos masses and mixing, that strongly suggest that the SM is not the ultimate theory of nature. In this context, we may need to add new scalars, to play the role of the DM candidate or in order to justify the difference between the mass scale of the neutrinos and the charged leptons. The question is, if there are more scalar doublets, how many of them? The simplest case is to add one more doublet. This is well motivated because it allows spontaneous $C P$ violation if at the same time

\footnotetext{
* Corresponding author.

E-mail addresses: ana@ift.unesp.br (A.C.B. Machado), vicente@ift.unesp.br (V. Pleitez).
} 
flavor changing neutral currents (FCNC) are allowed [3,4]. The latter processes strongly constrain the masses and the mixing angles in the scalar sectors. For a recent review of the phenomenology of the two Higgs doublet models (2HDM) see Ref. [5]. The next simple situation is having three doublets in which it is possible to have spontaneous and hard $C P$ violation [6] and at the same time to avoid FCNC if some extra symmetries are introduced. Next, we can introduce more Higgs scalar doublets, for instance, it may be motivated by the implementation of the Peccei-Quinn symmetry and the unification of the three interactions, see Ref. [7] and references therein.

Among all these possibilities the case of three doublets with the same quantum number is interesting if we assume that the replica of three generations occurs not only in the fermion sector but also in the scalar sector. However, a general three doublet model (3HDM) has a very complicated scalar potential with six parameters with dimension of mass $\left(\mu^{2} s\right)$, and many dimensionless ones $(\lambda s)$. Notwithstanding in physics, when the degrees of freedom augment it motivates the introduction of new symmetries. In fact, to reduce the number of parameters in the scalar sector usually symmetries, like $\mathbb{Z}_{2}$ [8], are introduced. In some cases one of the scalar doublets is inert. However, the $Z_{2}$ symmetry still allows four $\mu^{2}$ s and 23 dimensionless real parameters. The possibility of an $S_{3}$ symmetry is also explored in $[9,10]$. In the case of the $3 \mathrm{HDM}$ with $S_{3}$ symmetry has only two parameters with dimension of mass and eight dimensionless ones i.e., in terms of the number of parameters the scalar potential in the 3HDM plus a $S_{3}$ symmetry has almost the same as the general 2HDM. The problem with this model is that in general several possibilities are allowed, and some of them are not physical because they imply the existence of massless physical neutral scalar. Another difficulty is the existence of FCNC effects [11]. Usually also the neutral scalar with mass of $125 \mathrm{GeV}$ is obtained only in the decoupling limit [10]. All these effects arise mainly because, (i) the mass matrices mix all the scalars in each charge sector and, because of this, the unitary matrices that diagonalize the respective mass matrices are general ones in each case; (ii) an arbitrary vacuum alignment is assumed with all the vacuum expectation values (VEVS) being different.

Here we will consider a 3HDM with an $S_{3}$ symmetry in which the SM-like scalar is automatically identified without requiring a decoupling limit. This is a consequence of a particular vacuum alignment and the absence of FCNC at tree level is a consequence of this vacuum alignment plus the condition of fermions transforming trivially under the symmetry $S_{3}$. Another important consequence of this vacuum alignment is that the scalars in the $S_{3}$ doublet are inert $[12,13]$. It means that they do not contribute to the spontaneous symmetry breaking and do not couple to fermions. They interact only with the vector and the other scalar bosons.

The SM extensions with one inert doublet model (IDM) as a candidate to dark matter have been already considered in Refs. [14-22]. However having two inert doublets allows to have a multicomponent dark matter scenario [23-25], because not only the real scalar and pseudoscalar may be DM as in the IDM, but now we have two real scalar fields and two pseudoscalar ones, each of them may contribute to the DM density.

The outline of this paper is as follows. In Section 2 we give the most general scalar potential involving three Higgs doublets which is invariant under the gauge and $S_{3}$ symmetries. We also consider in that section the mass spectra in the scalar sector when all VEVs satisfy the alignment $v_{1}=v_{2}=v_{3}=$ $v=v_{S M} / \sqrt{3}$ and the singlet $(S)$ and the doublet $(D)$ of $S_{3}$ are originated from a triplet i.e., in the reducible representation. In this situation there are mass degenerate states in each scalar sector because a residual $S_{2}$ symmetry remains. However the mass degeneracy may be lifted by introducing terms that break the $S_{2}$ symmetry softly. We dubbed this case A. In Section 4 we consider the case when $S=H_{1}$ and $D=\left(H_{2}, H_{3}\right)$ with $v_{1}=v_{S M}$ and $v_{2}=v_{3}=0$. We call this case B. We show that both cases, before the spontaneous symmetry breaking (SSB), are related by a weak basis transformation. However, after the SSB both cases are still equivalent but only the vacuum alignment is considered here. We also consider in this case the situation when the $S_{2}$ symmetry is softly broken avoiding the mass degeneracy, in this case the equivalence between both cases is also lost. The Yukawa interactions are the same in both cases and are briefly discussed in Section 5. In Section 6 we study the positivity of the scalar potential at the tree level, while in Section 7 we consider some phenomenological consequences. Our conclusions are in Section 8 and in the appendices we show the constraint equations for arbitrary VEVs, for case A in Appendix A, and for case B in Appendix B. 


\section{Three Higgs-scalar doublet model and $S_{3}$ symmetry}

We present an extension of the electroweak standard model with three Higgs scalars, all of them transforming as doublets under $S U(2)$ and having $Y=+1$. Some of them transform under $S_{3}$ as a doublet $D=\left(D_{1}, D_{2}\right) \equiv \mathbf{2}$, and some as a singlet $S \equiv \mathbf{1}$. As we will see, the latter one is identified with the SM-like Higgs and the former ones are inert.

The most general scalar potential invariant under $S U(2) \otimes U(1)_{Y} \otimes S_{3}$ symmetry is given by:

$$
\begin{aligned}
V(D, S)= & \mu_{s}^{2} S^{\dagger} S+\mu_{d}^{2}\left[D^{\dagger} \otimes D\right]_{1}+\lambda_{1}\left(\left[D^{\dagger} \otimes D\right]_{1}\right)^{2}+\lambda_{2}\left[\left(D^{\dagger} \otimes D\right)_{1^{\prime}}\left(D^{\dagger} \otimes D\right)_{1^{\prime}}\right] \\
& +\lambda_{3}\left[\left(D^{\dagger} \otimes D\right)_{2^{\prime}}\left(D^{\dagger} \otimes D\right)_{2^{\prime}}\right]_{1}+\lambda_{4}\left(S^{\dagger} S\right)^{2}+\lambda_{5}\left[D^{\dagger} \otimes D\right]_{1} S^{\dagger} S \\
& +\left[\lambda_{6}\left[\left[S^{\dagger} D\right]_{2^{\prime}}\left[S^{\dagger} D\right]_{2^{\prime}}\right]_{1}+H . c .\right]+\lambda_{7} S^{\dagger}\left[D \otimes D^{\dagger}\right]_{1} S \\
& +\left[\lambda_{8}\left[\left(S^{\dagger} \otimes D\right)_{2^{\prime}}\left(D^{\dagger} \otimes D\right)_{2^{\prime}}\right]_{1}+\text { H.c. }\right] .
\end{aligned}
$$

Denoting an arbitrary doublet by $\mathbf{2}=\left(x_{1}, x_{2}\right)$, we have the product rule $\mathbf{S}$ as $\mathbf{2} \otimes \mathbf{2}=\mathbf{1} \oplus \mathbf{1}^{\prime} \oplus \mathbf{2}^{\prime}$ where $\mathbf{1}=x_{1} y_{1}+x_{2} y_{2}, \mathbf{1}^{\prime}=x_{1} y_{2}-x_{2} y_{1}, \mathbf{2}^{\prime}=\left(x_{1} y_{2}+x_{2} y_{1}, x_{1} y_{1}-x_{2} y_{2}\right)$, and $\mathbf{1}^{\prime} \otimes \mathbf{1}^{\prime}=\mathbf{1}$ [26]. Let us define $S=\left(s^{+} s^{0}\right)^{T}, D_{i}=\left(d_{i}^{+} d_{i}^{0}\right)^{T}, i=1$, 2. In terms of the $S$ and $D_{i}$ fields, the potential in Eq. (1) is written as

$$
\begin{aligned}
V\left(S, D_{1}, D_{2}\right)= & \mu_{s}^{2} S^{\dagger} S+\mu_{d}^{2}\left(D_{1}^{\dagger} D_{1}+D_{2}^{\dagger} D_{2}\right)+\lambda_{1}\left(D_{1}^{\dagger} D_{1}+D_{2}^{\dagger} D_{2}\right)^{2}+\lambda_{2}\left(D_{1}^{\dagger} D_{2}-D_{2}^{\dagger} D_{1}\right)^{2} \\
& +\lambda_{3}\left[\left(D_{1}^{\dagger} D_{2}+D_{2}^{\dagger} D_{1}\right)^{2}+\left(D_{1}^{\dagger} D_{1}-D_{2}^{\dagger} D_{2}\right)^{2}\right]+\lambda_{4}\left(S^{\dagger} S\right)^{2}+\lambda_{5}\left(D_{1}^{\dagger} D_{1}+D_{2}^{\dagger} D_{2}\right) S^{\dagger} S \\
& +\left[\lambda_{6}\left(S^{\dagger} D_{1} S^{\dagger} D_{1}+S^{\dagger} D_{2} S^{\dagger} D_{2}\right)+H . c .\right]+\lambda_{7} S^{\dagger}\left(D_{1} D_{1}^{\dagger}+D_{2} D_{2}^{\dagger}\right) S \\
& +\lambda_{8}\left[S^{\dagger} D_{1}\left(D_{1}^{\dagger} D_{2}+D_{2}^{\dagger} D_{1}\right)+S^{\dagger} D_{2}\left(D_{1}^{\dagger} D_{1}-D_{2}^{\dagger} D_{2}\right)+\text { H.c. }\right] .
\end{aligned}
$$

Notice that the potential is written in terms of the symmetry eigenstates independently of how we form the singlet and the doublet. If $\mu_{d}^{2}>0$ only the singlet $S$ gain a VEV and if $\lambda_{8}=0$ this vacuum is stable at tree and the one-loop level. For this term be forbidden we impose a $\mathbb{Z}_{2}$ symmetry under which $D \rightarrow-D$ and $S$ and all the other fields are even. However, in the appendix we consider the constraint equations with a general vacuum alignment in order to study under what conditions we have $\langle D\rangle=0$ and we find that independently of the sign of $\mu_{d}^{2}$, it is possible to have the vacuum alignment considered in this paper. The three-Higgs scalar potential has already been considered in the literature in Refs. $[9,10,27,28]$ but not in the inert doublets context. In fact, unlike the present paper, all these articles have used the $S_{3}$ symmetry to address the texture of the fermion mass matrices using a general vacuum alignment.

\section{Case $A$}

Let us now consider the case when the three scalar doublets are in the reducible triplet representation of $S_{3}$, say, $\mathbf{3}=\left(H_{1}, H_{2}, H_{3}\right)$ where $H_{i}=\left(H_{i}^{+} H_{i}^{0}\right)^{T}$. This reducible representation is broken down to the irreducible singlet and doublet ones, i.e., $\mathbf{3}=\mathbf{2}+\mathbf{1} \equiv D+S$, where:

$$
\begin{aligned}
& S=\frac{1}{\sqrt{3}}\left(H_{1}+H_{2}+H_{3}\right) \sim \mathbf{1}, \\
& D \equiv\left(D_{1}, D_{2}\right)=\left[\frac{1}{\sqrt{6}}\left(2 H_{1}-H_{2}-H_{3}\right), \frac{1}{\sqrt{2}}\left(H_{2}-H_{3}\right)\right] \sim \mathbf{2},
\end{aligned}
$$

or, explicitly in terms of the symmetry eigenstates $H_{i}^{+, 0}, i=1,2,3$

$$
\begin{aligned}
& S \equiv\left(\begin{array}{l}
s^{+} \\
s^{0}
\end{array}\right)=\frac{1}{\sqrt{3}}\left(\begin{array}{c}
H_{1}^{+}+H_{2}^{+}+H_{3}^{+} \\
H_{1}^{0}+H_{2}^{0}+H_{3}^{0}
\end{array}\right), \quad D_{1} \equiv\left(\begin{array}{c}
d_{1}^{+} \\
d_{1}^{0}
\end{array}\right)=\frac{1}{\sqrt{6}}\left(\begin{array}{c}
2 H_{1}^{+}-H_{2}^{+}-H_{3}^{+} \\
2 H_{1}^{0}-H_{2}^{0}-H_{3}^{0}
\end{array}\right), \\
& D_{2} \equiv\left(\begin{array}{c}
d_{2}^{+} \\
d_{2}^{0}
\end{array}\right)=\frac{1}{\sqrt{2}}\left(\begin{array}{c}
H_{2}^{+}-H_{3}^{+} \\
H_{2}^{0}-H_{3}^{0}
\end{array}\right) .
\end{aligned}
$$

The decomposition of the symmetry eigenstates we make as usual, as $H_{i}^{0}=(1 / \sqrt{2})\left(v_{i}+\eta_{i}^{0}+i a_{i}^{0}\right)$, $i=1,2,3$. We assume for the sake of simplicity that the VEVs are real, however see Section 7 . 
The general constraint equations for the case when all VEVs are different from zero are given in the Appendix A, Eq. (A.2). When $v_{1}=v_{2}=v_{3}=v$ these constraint equations are reduced to a simple equation:

$$
t_{1}=t_{2}=t_{3}=v\left(\mu_{s}^{2}+3 \lambda_{4} v^{2}\right),
$$

and if $t_{i}=0$ we have $\mu_{s}^{2}=-3 \lambda_{4} v^{2}=-\lambda_{4} v_{S M}^{2}<0$, which implies that $\lambda_{4}>0$.

All scalar mass square matrices have the form

$$
M_{n}^{2}=\left(\begin{array}{lll}
a_{n} & b_{n} & b_{n} \\
b_{n} & a_{n} & b_{n} \\
b_{n} & b_{n} & a_{n}
\end{array}\right),
$$

where $a_{n}, b_{n}>0$ and $n$ denotes the scalar sector: $n=h, a, c$ for the scalar, pseudo-scalar and charged scalar fields, respectively.

This type of matrix is diagonalized by an orthogonal matrix, $U_{T B M}: U_{T B M}^{T} M_{n}^{2} U_{T B M}=\operatorname{diag}\left(a_{n}+\right.$ $\left.2 b_{n}, a_{n}-b_{n}, a_{n}-b_{n}\right)$, with $a_{n}+2 b_{n} \geq 0$ and $a_{n}-b_{n} \geq 0, \forall n$, the $U_{T B M}$ is given by

$$
U_{T B M}=\left(\begin{array}{ccc}
\frac{1}{\sqrt{3}} & -\sqrt{\frac{2}{3}} & 0 \\
\frac{1}{\sqrt{3}} & \frac{1}{\sqrt{6}} & -\frac{1}{\sqrt{2}} \\
\frac{1}{\sqrt{3}} & \frac{1}{\sqrt{6}} & \frac{1}{\sqrt{2}}
\end{array}\right) .
$$

In the case of $C P$-even neutral scalars, we have $3 a_{h}=2 \mu_{d}^{2}+\left(2 \lambda_{4}+\bar{\lambda}^{\prime}\right) v_{S M}^{2}$, and $6 b_{h}=-2 \mu_{d}^{2}+$ $\left(4 \lambda_{4}-\bar{\lambda}^{\prime}\right) v_{S M}^{2}$, where $\bar{\lambda}^{\prime}=\left(\lambda_{5}+\lambda_{7}+2 \lambda_{6}\right)$, and the eigenvalues are the following:

$$
\begin{aligned}
& m_{h_{1}}^{2} \equiv m_{h}^{2}=2 \lambda_{4} v_{S M}^{2}, \\
& m_{h_{2}}^{2}=m_{h_{3}}^{2} \equiv m_{H}^{2}=\mu_{d}^{2}+\frac{1}{2} \bar{\lambda}^{\prime} v_{S M}^{2}=\mu_{d}^{2}+\frac{1}{4} \frac{\bar{\lambda}^{\prime}}{\lambda_{4}} m_{h}^{2},
\end{aligned}
$$

where we have used $v=v_{S M} / \sqrt{3}$.

Denoting as $h_{i}^{0}$ the mass eigenstates, we have $h_{i}^{0}=\sum_{i}\left(U_{T B M}^{T}\right)_{i j} \eta_{j}^{0}$, where $U_{T B M}$ is given in (7). Explicitly we have

$$
\left(\begin{array}{l}
h^{0} \\
h_{2}^{0} \\
h_{3}^{0}
\end{array}\right)=\left(\begin{array}{c}
\frac{1}{\sqrt{3}}\left(\eta_{1}^{0}+\eta_{2}^{0}+\eta_{3}^{0}\right) \\
-\frac{1}{\sqrt{6}}\left(2 \eta_{1}^{0}-\eta_{2}^{0}-\eta_{3}^{0}\right) \\
-\frac{1}{\sqrt{2}}\left(\eta_{2}^{0}-\eta_{3}^{0}\right)
\end{array}\right) \equiv \operatorname{Re}\left(\begin{array}{c}
s^{0} \\
-d_{1}^{0} \\
-d_{2}^{0}
\end{array}\right),
$$

and the scalar $h^{0} \equiv \operatorname{Re} s^{0}$ which, in Section 5, will be identified with the SM Higgs boson and the doublet $h_{1} \equiv h$ with the SM scalar doublet.

In the $C P$-odd neutral scalars sector, the mass matrix is given as in Eq. (6) but now with $3 a_{a}=$ $2 \mu_{d}^{2}+\bar{\lambda}^{\prime \prime} v_{S M}^{2}$ and $6 b_{a}=-2 \mu_{d}^{2}-\bar{\lambda}^{\prime \prime} v_{S M}^{2}$, where $\bar{\lambda}^{\prime \prime}=\left(\lambda_{5}+\lambda_{7}-2 \lambda_{6}\right)$ and in this case we obtain the following masses:

$$
\begin{aligned}
& m_{A_{1}}^{2}=0, \\
& m_{A_{2}}^{2}=m_{A_{3}}^{2} \equiv m_{A}^{2}=\mu_{d}^{2}+\frac{1}{2} \bar{\lambda}^{\prime \prime} v_{S M}^{2}=\mu_{d}^{2}+\frac{1}{4} \frac{\bar{\lambda}^{\prime \prime}}{\lambda_{4}} m_{h}^{2} .
\end{aligned}
$$

Denoting $A_{i}^{0}$ the pseudo-scalar mass eigenstates, we have $A_{i}^{0}=\sum_{i}\left(U_{T B M}^{T}\right)_{i j} a_{j}^{0}$ and making the same as in Eq. (9) we obtain that $A^{0}=\operatorname{Im} s^{0}$ is the would-be Goldstone boson, while $A_{2}^{0}=-\operatorname{Im} d_{1}^{0}$ and $A_{3}^{0}=-\operatorname{Im} d_{2}^{0}$ are physical $C P$ odd fields. 
Similarly in the charged scalars sector we use Eq. (6) with $6 a_{c}=2 \mu_{d}^{2}+\lambda_{5} v_{S M}^{2}$ and $12 b_{c}=$ $-2 \mu_{d}^{2}-\lambda_{5} v_{S M}^{2}$ and in this case we obtain the following masses:

$$
\begin{aligned}
& m_{c_{1}}^{2}=0, \\
& m_{c_{2}}^{2}=m_{c_{3}}^{2} \equiv 2 m_{c}^{2}=\mu_{d}^{2}+\frac{\lambda_{5}}{2} v_{S M}^{2}=\mu_{d}^{2}+\frac{1}{8} \frac{\lambda_{5}}{\lambda_{4}} m_{h}^{2},
\end{aligned}
$$

and, if $H_{i}^{+}$denote the charged scalar symmetry eigenstates and $h_{i}^{+}$the respective mass eigenstates, we have $h_{i}^{+}=\sum_{i}\left(U_{T B M}^{T}\right)_{i j} H_{j}^{+}$. Using again the Eq. (7) we obtain $s^{+}=h^{+}$the would-be charged Goldstone boson, and the physical charged scalars: $-d_{1}^{+}=h_{2}^{+}$and $-d_{2}^{+}=h_{3}^{+}$.

We summarize these results by using mixing matrix in Eq. (7), and writing the Higgs scalars doublet $D$ and the singlet $S$, but now in terms of the mass eigenstates, $h_{i}^{0}, A_{i}^{0}$ and $h_{i}^{ \pm}$, as

$$
S \equiv \phi=\left(\begin{array}{c}
h^{+} \\
\frac{1}{\sqrt{2}}\left(\sqrt{3} v+h^{0}+i A^{0}\right)
\end{array}\right), \quad D \equiv-\left(\phi_{1}, \phi_{2}\right), \quad \phi_{k}=\left(\begin{array}{c}
h_{k}^{+} \\
\frac{1}{\sqrt{2}}\left(h_{k}^{0}+i A_{k}^{0}\right)
\end{array}\right),
$$

where $k=2,3$.

We have the sum rule from Eqs. (8), (10) and (11):

$$
m_{H}^{2}+m_{A}^{2}+2 m_{c}^{2}=3 \mu_{d}^{2}+\frac{1}{\lambda_{4}}\left(\bar{\lambda}^{\prime}+\bar{\lambda}^{\prime \prime}+\lambda_{5}\right) m_{h}^{2} .
$$

The mass degeneracy in Eqs. (8), (10) and (11), is due to a residual symmetry as we will see below. The $\mu_{d}^{2}$ parameter appearing in these equations is not related to the spontaneous symmetry breaking. Thus, since $\mu_{d}^{2}$ is not protected by any symmetry, it may be larger than the electroweak scale. On one hand, if $\mu_{d}^{2}>v_{S M}^{2}$ (assuming the $\lambda^{\prime} s$ are of order one) the masses of the scalar $h_{2,3}^{0}$, pseudo-scalar $A_{2,3}^{0}$ and the charged scalar $h_{2,3}^{ \pm}$are heavier than $h^{0}$, independently of the values of the $\lambda^{\text {'s }}$ and $v_{S M}$. On the other hand, if $\mu_{d}^{2}<0$ and $\bar{\lambda}^{\prime}, \bar{\lambda}^{\prime \prime}>0, \lambda_{5}>0$, all these particles may be lighter than $h^{0}$. However, since $\lambda_{5}$ may be negative, $\lambda_{4}$ is always positive, and in this case $m_{c}^{2}<0$, as can be seen from Eq. (11), here we will consider only $\mu_{d}^{2}>0$ and larger than $\left|\left(\lambda_{5} / 8 \lambda_{4}\right)\right| m_{h}^{2}$.

The potential in Eq. (2) can be written in terms of $S U(2)$ scalar doublets with their components being the mass eigenstates given in Eq. (12):

$$
\begin{aligned}
V\left(\phi_{i}\right)= & 3 \lambda_{4} v^{2} \phi^{\dagger} \phi+\mu_{d}^{2}\left(\phi_{1}^{\dagger} \phi_{1}+\phi_{2}^{\dagger} \phi_{2}\right)+\lambda_{1}\left(\phi_{1}^{\dagger} \phi_{1}+\phi_{2}^{\dagger} \phi_{2}\right)^{2}+\lambda_{2}\left(\phi_{1}^{\dagger} \phi_{2}-\phi_{2}^{\dagger} \phi_{1}\right)^{2} \\
& +\lambda_{3}\left[\left(\phi_{1}^{\dagger} \phi_{2}+\phi_{2}^{\dagger} \phi_{1}\right)^{2}+\left(\phi_{1}^{\dagger} \phi_{1}-\phi_{2}^{\dagger} \phi_{2}\right)^{2}\right]+\lambda_{4}\left(\phi^{\dagger} \phi\right)^{2}+\lambda_{5} \phi^{\dagger} \phi\left(\phi_{1}^{\dagger} \phi_{1}+\phi_{2}^{\dagger} \phi_{2}\right) \\
& +\lambda_{7}\left[\left|\phi^{\dagger} \phi_{1}\right|^{2}+\left|\phi^{\dagger} \phi_{2}\right|^{2}\right]+\left\{\lambda_{6}\left[\left(\phi^{\dagger} \phi_{1}\right)^{2}+\left(\phi_{2}^{\dagger} \phi\right)^{2}\right]+\lambda_{8}\left[\phi^{\dagger} \phi_{1}\left(\phi_{1}^{\dagger} \phi_{2}+\phi_{2}^{\dagger} \phi_{1}\right)\right.\right. \\
& \left.\left.+\phi^{\dagger} \phi_{2}\left(\phi_{2}^{\dagger} \phi_{2}-\phi_{1}^{\dagger} \phi_{1}\right)\right]+ \text { H.c. }\right\} .
\end{aligned}
$$

Notice that this scalar potential is the same as that in Eq. (2). However the earlier one was written in terms of the symmetry eigenstates and (14) is in terms on the mass eigenstates. This occurs only in this model and not in any 3HDM and it is a consequence of the $S_{3}$ symmetry and the vacuum alignment.

Notice that this scalar potential with three Higgs scalar doublets under $S U(2)$, is as simple as the two doublet case, see for instance in Ref. [29]. From Eq. (14), we can see that if $\lambda_{8}=0$ there is still a residual $S_{2}$ symmetry: it is invariant under the exchange of the doublets $\phi_{1} \leftrightarrow \phi_{2}$. Notice, however, that the mass degeneracy is due to the fact that the $\lambda_{8}$ term does not contribute to the Higgs scalar masses, this is easy to be verified, once $\lambda_{8}$ corresponds only to the trilinear and quartic interactions among the three doublets. Anyway, we have considered only the $\lambda_{8}=0$ case due to the $\mathbb{Z}_{2}$ symmetry considered above.

We will show later on under which conditions the potential in Eq. (2) (or (14)) is bounded from below. For the moment, just notice that when $v_{1}=v_{2}=v_{3}$, if $\lambda_{4}>0$, the minimum of the scalar potential $\left(V_{\min }=-\lambda_{4} v_{S M}^{4}\right)$ is global and stable minimum if the masses square, given in (8), (10) and 
(11) are all positive and, if the conditions for the $\lambda$ 's given in Section 6 are satisfied. However, the stability of the solution $v_{1}=v_{2}=v_{3}$ under radiative corrections will be studied elsewhere.

The residual $S_{2}$ symmetry can be broken, if necessary, to avoid the mass degeneracy and also the domain wall problem. This can be done by quantum corrections [30] and/or by soft terms in the scalar potential. As an illustration, here we break this symmetry by adding the following quadratic terms $\mu_{n m}^{2} H_{n}^{\dagger} H_{m}, n, m=2,3$ to the scalar potential in (1). The mass matrices in all the scalar sectors are now of the form

$$
M_{n}^{2}=\left(\begin{array}{ccc}
a_{n} & b_{n} & b_{n} \\
b_{n} & a_{n}+\mu_{22}^{2} & b_{n}+\mu_{23}^{2} \\
b_{n} & b_{n}+\mu_{23}^{2} & a_{n}+\mu_{33}^{2}
\end{array}\right),
$$

where $\mu_{n m}^{2}$ are naturally small, and we will assume that are real for the sake of simplicity. Although when $\mu_{22}^{2}=\mu_{33}^{2}=v^{2}$ and $\mu_{23}^{2}=\mu^{2}$ the matrix above is still diagonalized by the tribimaximal matrix, as the neutrinos masses [31], this is not possible with scalars fields: in this case there is no would-be Goldstone bosons. In order to have the correct number of these bosons we have to impose that $\mu_{22}^{2}=\mu_{33}^{2}=-\mu_{23}^{2} \equiv \mu^{2}$. In this case the matrix in Eq. (15) is still diagonalized by tribimaximal matrix in Eq. (7), and the eigenvalues are now $\left(2 a_{n}+b_{n}, a_{n}-b_{n}, a_{n}-b_{n}+\mu^{2}\right)$ and we still have $S=\phi$ and $D=-\left(\phi_{1}, \phi_{2}\right)$, as in the previous case.

\section{A change of weak basis: Case $B$}

We can build the singlet and a doublet of $S_{3}$ with just one $S U(2)$-doublet, say $H_{1}$, and the other two, say $\mathrm{H}_{2}$ and $\mathrm{H}_{3}$, transform as the irreducible doublet of $S_{3}$, i.e.,

$$
S=H_{1} \sim \mathbf{1}, \quad D=\left(H_{2}, H_{3}\right) \sim \mathbf{2} .
$$

But, note that, the two bases are related by the tribimaximal matrix in Eq. (7), i.e,

$$
\left(\begin{array}{c}
S \\
D_{1} \\
D_{2}
\end{array}\right)=U_{\text {TBM }}^{T}\left(\begin{array}{l}
H_{1} \\
H_{2} \\
H_{3}
\end{array}\right)
$$

with $U_{T B M}^{T}$ being the transpose of the matrix in Eq. (7) and $S, D_{1}$ and $D_{2}$ here are those in Eq. (3). The representation in Eq. (16), is called here case B. It was considered since a long time ago [27,32-35] but in other context and different motivations.

Although both cases in (3) and (16) are related by the transformation in Eq. (17) and can be considered just the same model in two different basis, we can see that this is true only before the spontaneous symmetry breaking. The VEV of the $S_{3}$ triplet in case $\mathrm{A}$ is given by

$$
\left\langle\left(\begin{array}{l}
H_{1} \\
H_{2} \\
H_{3}
\end{array}\right)\right\rangle=\left(\begin{array}{l}
v_{1} \\
v_{2} \\
v_{3}
\end{array}\right) .
$$

When the decomposition in Eq. (3) is used and the vacuum alignment $v_{1}=v_{2}=v_{3}=\frac{v_{S M}}{\sqrt{3}}$ is used it implies the inert character of the doublet $D$. However, in case B we have

$$
\left\langle\left(\begin{array}{l}
H_{1} \\
H_{2} \\
H_{3}
\end{array}\right)\right\rangle=\left(\begin{array}{c}
v_{S M} \\
0 \\
0
\end{array}\right) .
$$

We see that the vacua in (18) and (19) are related by the transformation in (17) only when $v_{1}=$ $v_{2}=v_{3}=\frac{v_{S M}}{\sqrt{3}}$. Hence, only in this situation both cases are identical before and after the spontaneous symmetry breaking. But in a general vacuum the inert character of the doublet is lost because in this case the mass matrices are of the general form, a full $3 \times 3$ matrix, and after the field rotation the SMHiggs like will be a linear combination of this three fields, this implies that at tree level and/or loop level all scalars couple to all fermions. Hence we have to impose in case B that $v_{2}=v_{3}=0$. In fact, the constraint equations are different and are given in the Appendix B. These constraint equations are the same, see Eq. (5) with $3 v^{2}=v_{S M}^{2}$ only in the vacuum alignment used in this paper. 
The constraint equations in Eq. (B.2) implies, with the vacuum alignment given above, $\mu_{s}^{2}=$ $-\lambda_{4} v_{S M}^{2}$ and the mass square matrices are all diagonal: there is no mixing among the scalar fields in each charge sector. At tree level the masses are the same as in case A, see Eqs. (8)-(11). The doublets of $S U$ (2) written in terms of the mass eigenstates are denoted, as before, by $\phi$ and $\phi_{1,2}$. In this case the scalar potential in terms of these fields is given also in Eq. (14) again with $\lambda_{8}=0$, this shows that even after the SSB the models are equivalent. Unlike the case A, there is no mixing among the mass eigenstate scalar fields therefore these fields are in the irreducible representations of $S_{3}$ too: $S \equiv \phi$ and $D=\left(d_{1}, d_{2}\right) \equiv\left(\phi_{1}, \phi_{2}\right)$.

The transformation $\phi_{1} \leftrightarrow \phi_{2}$ is again a residual $S_{2}$ symmetry which, if necessary, can be softly broken by adding terms like $\mu^{2} \mathrm{H}_{2}^{\dagger} H_{3}$, ( $\mu^{2}$ is also considered to be real for simplicity). In this case, the mass matrices are given by

$$
M_{n}^{2}=\left(\begin{array}{ccc}
m_{n_{1}}^{2} & 0 & 0 \\
0 & m_{n_{2}}^{2} & \mu^{2} \\
0 & \mu^{2} & m_{n_{2}}
\end{array}\right),
$$

where $n=h, a, c$, for scalar, pseudo-scalar and charged scalar field, respectively. The mixing now is only in the inert sector and the masses square are

$$
\begin{array}{lll}
\bar{m}_{h_{1}}^{2}=m_{h}^{2}, & \bar{m}_{h_{2}}^{2}=m_{h}^{2}-\mu^{2}, & \bar{m}_{h_{3}}^{2}=m_{h}^{2}+\mu^{2}, \\
\bar{m}_{a_{1}}^{2}=0, & \bar{m}_{a 2}^{2}=m_{a}^{2}-\mu^{2}, & \bar{m}_{a 3}^{2}=m_{a}^{2}+\mu^{2}, \\
\bar{m}_{c_{1}}^{2}=0, & \bar{m}_{c_{2}}^{2}=m_{c}^{2}-\mu^{2}, & \bar{m}_{c_{3}}^{2}=m_{c}^{2}+\mu^{2},
\end{array}
$$

where $m_{h}^{2}, m_{a}^{2}$ and $m_{c}^{2}$ are given in Eqs. (8), (10) and (11), respectively. The mass matrices of the form in (20) are diagonalized by the orthogonal matrix

$$
U=\left(\begin{array}{ccc}
1 & 0 & 0 \\
0 & -\frac{1}{\sqrt{2}} & \frac{1}{\sqrt{2}} \\
0 & \frac{1}{\sqrt{2}} & \frac{1}{\sqrt{2}}
\end{array}\right),
$$

and the mixing between $\phi_{1}$ and $\phi_{2}$ is maximal.

Thus, in terms of the mass eigenstate fields, the scalar doublets of $S U(2)$ are written as $S=\phi$ and $D \equiv-\left(D_{1}, D_{2}\right)=-\left(-\phi_{1}+\phi_{2}, \phi_{1}+\phi_{2}\right)$, where $\phi_{i}$ are the $S U(2)$ doublets written in terms of the mass eigenstate fields. Explicitly

$$
\begin{aligned}
& S \equiv \phi=\left(\begin{array}{c}
h^{+} \\
\frac{1}{\sqrt{2}}\left(v_{S M}+h^{0}+i A^{0}\right)
\end{array}\right), \\
& \phi_{1}=\left(\begin{array}{c}
\frac{1}{\sqrt{2}}\left(-h_{2}^{+}+h_{3}^{+}\right) \\
\frac{1}{2}\left[-h_{2}^{0}+h_{3}^{0}+i\left(-A_{2}^{0}+A_{3}^{0}\right)\right]
\end{array}\right), \quad \phi_{2}=\left(\begin{array}{c}
\frac{1}{\sqrt{2}}\left(h_{2}^{+}+h_{3}^{+}\right) \\
\frac{1}{2}\left[h_{2}^{0}+h_{3}^{0}+i\left(A_{2}^{0}+A_{3}^{0}\right)\right]
\end{array}\right) .
\end{aligned}
$$

It is important to note again that after the degeneracy breaking we lose the connection between the two cases as can be seen by comparing Eq. (12) with (23).

\section{The Yukawa sector}

If in cases $\mathrm{A}$ and $\mathrm{B}$ in the lepton and quark sectors all fields transform as singlet under $S_{3}$, they only interact with the singlet $S$ as following:

$$
-\mathcal{L}_{\text {yukawa }}=\bar{L}_{i L}\left(G_{i j}^{l} l_{j R} S+G_{i j}^{v} v_{j R} \tilde{S}\right)+\bar{Q}_{i L}\left(G_{i j}^{u} u_{j R} \tilde{S}+G_{i j}^{d} d_{j R} S\right)+\text { H.c. }
$$

$\tilde{S}=i \tau_{2} S^{*}$ and we have included right-handed neutrinos. 
We see that the fermion masses, as in the SM, arise only through the VEV of the singlet $S$ which is the only field, or linear combination of fields, with a non-zero VEV, see Eqs. (12) and (23). Hence, there is no FCNC in the lepton and quark sectors at the tree level. Moreover, we obtain arbitrary mass matrices from Eq. (24), because there is just one source of the fermion masses which are given by $M^{f}=\left(v_{S M} / \sqrt{2}\right) G^{f}, f=l, v, u, d$ and where $v_{S M}=246 \mathrm{GeV}$. The neutral interactions are $\left(\sqrt{2} / v_{S M}\right) \bar{f}_{L} \hat{M}^{f} f_{R} h^{0}$, where $\hat{M}^{f}$ is the diagonal mass matrix in the $f$-sector. These mass matrices are general enough to accommodate a realistic $V_{P M N S}$ and $V_{C K M}$ mixing matrices. Moreover, since the righthanded neutrinos may have a Majorana mass term we can have a type-I seesaw mechanism.

Notwithstanding, unlike the case of the SM, having only one source of fermion masses is not guaranteed to avoid FCNC in the scalar sector. In fact, the case of natural flavor conservation when there are discrete symmetries was not considered in Ref. [36]. Hence, it is worth considering briefly this issue. Let $\mathscr{D}$ be a generic non-Abelian discrete symmetry with multiplication law $*$ under which the left- and right-handed fermions, namely $f_{\mathscr{D}_{L}}$ and $f_{\mathscr{D}_{R}}$, are in different representations of the gauge symmetry but are singlet under the $\mathscr{D}$ symmetry. The scalar multiplets, $H_{\mathscr{D}}$, transform non-trivially under the gauge symmetry, but are singlet of $\mathscr{D}$ since this is the scalar that couple to fermions. The Yukawa interactions are of the form $\bar{f}_{\mathscr{D}_{L}} * f_{\mathscr{D}_{R}} * H_{\mathscr{D}} \sim \mathbf{1}$, i.e., it is invariant under the gauge and discrete transformations. Even if $H_{\mathscr{D}}$ is in the trivial representation of $\mathscr{D}$ as we have assumed, without the vacuum alignment discussed above there are FCNC in each charge sector. With an arbitrary vacuum alignment the relation in Eq. (9), which implies that $\operatorname{Re} S^{0}=h^{0}$, is no longer valid and $S^{0}$ is a linear component of the three neutral mass eigenstates and all of them contribute to the fermion mass matrices. It suggests that the vacuum alignment can be added to the conditions in Ref. [36] to have natural flavor conservation in neutral currents at the tree level when discrete symmetries are present in the model. Here we have considered $\mathscr{D}=S_{3}$.

\section{Analysis of the scalar potential}

The scalar potential has to be bounded from below to ensure its stability. In the SM this is easy at least at tree level, we just have to ensure that the quartic term in the potential has $\lambda>0$. In theories that increase the number of scalar multiplets it is more difficult to ensure the positivity of the potential in all directions. A scalar potential has a quadratic form in the quartic couplings in the form $A_{a b} \xi_{a}^{2} \xi_{b}^{2}$. If the matrix $A_{a b}$ is copositive in the sense of Ref. [37], it is possible to ensure that the potential is bounded from below. Let us apply this analysis to our case.

We obtain the copositive conditions in the quartic terms in the scalar potential given in Eq. (14), by defining:

$$
\left|\zeta_{i}\right|^{2}=\xi_{i}^{2}, \quad \zeta_{i}^{\dagger} \zeta_{j}=\xi_{i} \xi_{j} \rho_{i} e^{i \theta_{i}}
$$

where $\zeta_{i}=S, D_{1}, D_{2}$, and $\rho_{i}$ and $\theta_{i}$ are not physical parameters. From the scalar potential of Eq. (2) we obtain the matrix $A$ in the base $\left(\xi_{1}^{2}, \xi_{2}^{2}, \xi_{3}^{2}\right)$ the matrix elements are given by:

$$
\begin{aligned}
& A_{11}=\lambda_{4}, \\
& A_{22}=\lambda_{1}+\lambda_{3}, \\
& A_{33}=A_{22}, \\
& A_{12}=A_{21}=\frac{1}{2}\left[\lambda_{5}+\rho_{1}^{2}\left(\lambda_{7}+2 \lambda_{6} \cos \left(2 \phi_{1}\right)\right)\right], \\
& A_{13}=A_{31}=\frac{1}{2}\left[\lambda_{5}+\rho_{2}^{2}\left(\lambda_{7}+2 \lambda_{6} \cos \left(2 \phi_{2}\right)\right)\right], \\
& A_{23}=A_{32}=2\left(\lambda_{3}-\lambda_{2}\right)+\rho_{3}^{2}\left(\lambda_{2}+\lambda_{3}\right) \cos \left(2 \phi_{3}\right) .
\end{aligned}
$$

Now we most minimize the potential with respect to the free parameters $\rho_{i}$ and $\phi_{i}$. For the terms $2 \lambda_{7} \cos \left(2 \phi_{1}\right)$ and $2 \lambda_{7} \cos \left(2 \phi_{2}\right)$ it is obvious that the minimum will be when $\cos \left(2 \phi_{1}\right)=\cos \left(2 \phi_{2}\right)=$ -1 , for the element $A_{23}$ to the minimum occurs for $\rho_{3}=1$ and $\cos \left(2 \phi_{3}\right)=-1$, which leaves us with

$$
\begin{aligned}
& A_{11}=\lambda_{4}, \\
& A_{22}=\lambda_{1}+\lambda_{3},
\end{aligned}
$$




$$
\begin{aligned}
& A_{33}=A_{22} \\
& A_{12}=A_{21}=\frac{1}{2}\left(\lambda_{5}+\rho_{1}^{2}\left(\lambda_{7}-2 \lambda_{6}\right)\right), \\
& A_{13}=A_{31}=\frac{1}{2}\left(\lambda_{5}+\rho_{2}^{2}\left(\lambda_{7}-2 \lambda_{6}\right)\right), \\
& A_{23}=A_{32}=-4 \lambda_{2} .
\end{aligned}
$$

Now if $\lambda_{7}-2 \lambda_{6} \geq 0$, the minimum of the potential is obtained by setting $\rho_{1}=\rho_{2}=0$, but if $\lambda_{7}-2 \lambda_{6} \leq 0$ then the minimum is given by $\rho_{1}=\rho_{2}=1$. To simplify our analysis and since the results for $\rho_{1}$ and $\rho_{2}$ are equal we will set $\rho_{1}=0$ and $\rho_{2}=1$. Finally we have the following expressions for the matrix elements,

$$
A=\left(\begin{array}{ccc}
\lambda_{4} & \frac{1}{2} \lambda_{5} & \frac{1}{2}\left[\lambda_{5}+\lambda_{7}-2 \lambda_{6}\right] \\
& \lambda_{1}+\lambda_{3} & -4 \lambda_{2} \\
& & \lambda_{1}+\lambda_{3}
\end{array}\right) .
$$

For a symmetric matrix $A$ of order 3 the copositivity criteria are summarized as follows: $a_{i i}>0$ and $v_{i j}=a_{i j}+\sqrt{a_{i i} a_{j j}}>0$ and $\sqrt{a_{11} a_{22} a_{33}}+a_{12} \sqrt{a_{33}}+a_{13} \sqrt{a_{22}}+a_{23} \sqrt{a_{11}}+\sqrt{v_{12} v_{13} v_{23}}>0$. Explicitly we obtain:

$$
\begin{aligned}
& \lambda_{4}>0, \\
& \lambda_{1}+\lambda_{3}>0, \\
& \lambda_{5}+2 \sqrt{\lambda_{4}\left(\lambda_{1}+\lambda_{3}\right)}>0, \\
& \lambda_{5}+\lambda_{7}-2 \lambda_{6}+2 \sqrt{\lambda_{4}\left(\lambda_{1}+\lambda_{3}\right)}>0, \\
& \lambda_{1}+\lambda_{3}>4 \lambda_{2},
\end{aligned}
$$

and

$$
\begin{aligned}
& \left(\lambda_{1}-2 \lambda_{2}-\lambda_{3}\right) \sqrt{\lambda_{4}}+\sqrt{\left(-4 \lambda_{2}\right)\left(\lambda_{1}+\lambda_{3}\right) \lambda_{4}} \\
& +\sqrt{\lambda_{1}+\lambda_{3}}\left(\lambda_{5}+\lambda_{7}-2 \lambda_{6}\right)+\frac{\sqrt{\lambda_{1}-\lambda_{2}}\left(2 \sqrt{\left(\lambda_{1}+\lambda_{3}\right) \lambda_{4}}+\lambda_{5}+\lambda_{7}-2 \lambda_{6}\right)}{\sqrt{2}}>0, \\
& \left(-4 \lambda_{2}\right) \sqrt{\lambda_{4}}+\sqrt{\left(-4 \lambda_{2}\right)\left(\lambda_{1}+\lambda_{3}\right) \lambda_{4}}+\sqrt{\lambda_{1}+\lambda_{3}}\left(\lambda_{5}\right) \\
& +\frac{\sqrt{\lambda_{1}-\lambda_{2}}\left(2 \sqrt{\left(\lambda_{1}+\lambda_{3}\right) \lambda_{4}}+\lambda_{5}\right)}{\sqrt{2}}>0 .
\end{aligned}
$$

It is easy to verify that if the conditions in Eqs. (29) are satisfied the conditions in Eq. (30) are automatically satisfied. Hence, the positivity of the scalar potential is guarantee just by the conditions in Eqs. (29).

\section{Some phenomenological consequences}

It is well known that two-Higgs doublet models have an interesting phenomenology [5]. For instance, (i) in a broad class of this type of models there is $C P$ violation arising purely from the exchange of Higgs bosons but FCNC are allowed [3], and (ii) in the class of models with inert scalars the lightest neutral fields is, at least in some range of the parameters, a dark matter candidate in the universe. Here we will consider only these two phenomenological aspects in this model. The first one is $C P$ violation and, secondly the possibility of having a dark matter candidate.

In general in three Higgs doublet models there is also $C P$ violation via de exchange of scalar fields [6]. We will analyze this issue in the present model. In case A, and the potential in Eq. (1) or in Eq. (2). Let us suppose that the VEVs are complex, and still imposing $v_{1} e^{i \theta_{1}}=v_{2} e^{i \theta_{2}}=v_{3} e^{i \theta_{3}}=V e^{i \Theta}$ as a stable minimum of the scalar potential. The phase $\Theta$, which appears only in the singlet $S$, can 
be transformed away with a global $U(1)$ transformation as it happens in the standard model. On the other hand, if $\theta_{1} \neq \theta_{2} \neq \theta_{3}$ we lost the inert feature of the two $S U$ (2) doublets in $D=\left(\phi_{1}, \phi_{2}\right)$. Thus, if we want two inert doublets there is no spontaneous $C P$ violation through the VEVs. We can also consider the possibility to have hard explicit $C P$ violation through complex coupling constants in the scalar potential because $\lambda_{6}$ may be complex, we can define $\lambda_{6}=\left|\lambda_{6}\right| e^{i \alpha_{6}}$. In this case, it is possible to transform away the $\lambda_{6}$ phase by making the global phase rotations $S \rightarrow S e^{i a_{S}}$ and $D \rightarrow D e^{i a_{D}}$, and choosing $a_{D}-a_{S}=\alpha_{6} / 2$, the $\lambda_{6}$ phase can be eliminated, wherefore we see that in this context there is no $C P$ violation in the scalar sector, just the hard violation in the quark and lepton mixing matrices.

We can try, also, to have soft explicit $C P$ violation through the quadratic non-diagonal term in the scalar potential $\mu^{2} h_{2}^{\dagger} h_{3}$ assuming that $\mu^{2}$ is complex, as in Ref. [4]. However it is not possible in case A once the mass matrices in Eq. (15) are not diagonalized by tribimaximal-type matrix and the inert feature of the two extra doublets is lost. However, this source of $C P$ violation is possible in case $B$ since, as can be seen from Eq. (20), notwithstanding the mixing and the $C P$ violation occurs only in the inert sector.

It is well known that there exists a range of the parameters in which an inert doublet is a candidate for dark matter (DM) [13-15,30]. This may also imply, in the present model, contributions to the invisible decay of the SM-like Higgs [38-40]. It has been shown in Ref. [22] that, in the context of one inert Higgs doublet (IDM), there are three allowed regions of masses that are compatible with observed value of $\Omega_{\mathrm{DM}} h^{2}$ : (i) $\lesssim 10 \mathrm{GeV}$; (ii) $40-150 \mathrm{GeV}$, and (iii) $\gtrsim 500 \mathrm{GeV}$. Notice that the regions (i) and that in $40-60 \mathrm{GeV}$ there is SM Higgs invisible decay.

The same may happen in the present model with $h_{2,3}^{0}$. Here we will only show that, for a range of the parameters and for the three allowed regions above the spin-independent cross section for the $h_{2,3}^{0}$-nucleon scattering agrees with the Xenon100 results [41], the Lux results [42] and the theoretical prediction of Xenon1T. And at the same time for the region (i) and $40-60 \mathrm{GeV}, h^{0} \rightarrow h_{2,3}^{0}$ may be compatible with the invisible width decay. Here we will consider only when there is mass degeneracy in case $\mathrm{A}$.

The spin-independent cross section for DM-nucleon scattering is given by [22]:

$$
\sigma_{S I}=2 \times \frac{m_{p}^{4} \bar{\lambda}^{\prime 2} f^{2}}{4 \pi\left(m_{p}+m_{h_{2}^{0}}\right)^{2} m_{h_{2}^{0}}^{4}}
$$

where the factor 2 is because we have two mass degenerated inert scalars, and $f=0.326$, see [39]; and the invisible Higgs width by:

$$
\Gamma\left(h^{0} \rightarrow h_{2}^{0} h_{2}^{0}\left(h_{3}^{0} h_{3}^{0}\right)\right)=2 \times \frac{\bar{\lambda}^{\prime 2} v_{S M}^{2}}{32 \pi m_{h_{2}^{0}}} \sqrt{1-\left(\frac{4 m_{h_{2}^{0}}}{m_{h}}\right)^{2}} .
$$

In Fig. 1 we show the excluded region given by Xenon100 [41] and Lux [42] results and the theoretical prediction for Xenon1T, Fig. 1(a) shows the behavior of Eq. (31) as a function of the masses for a fixed $\bar{\lambda}^{\prime}$ for masses less than $10 \mathrm{GeV}$, in this case the best solution is for $\bar{\lambda}^{\prime}=5 \times 10^{-4}$, but with masses lower than $6 \mathrm{GeV}$ all values are allowed. Fig. 1(b) shows the behavior of Eq. (31) as a function of $\bar{\lambda}^{\prime}$ for masses between 40 and $160 \mathrm{GeV}$, in this case we have two good solution for $\bar{\lambda}^{\prime}=5 \times 10^{-4}$ for the entire range and $\bar{\lambda}^{\prime}=10^{-3}$ for masses between 60 and $160 \mathrm{GeV}$. Finally in Fig. 1(c) we show that for masses larger than $500 \mathrm{GeV} \bar{\lambda}^{\prime}$ is allowed for a range between $5 \times 10^{-4}$ and $9 \times 10^{-3}$. These values are in agreement with the calculation of the relic density for this model as shown in Ref. [43].

In Fig. 2(a) we show the invisible Higgs width, using Eq. (32), and in Fig. 2(b) the branching ratio $\operatorname{Br}(h \rightarrow$ inv $)=\frac{\Gamma(h \rightarrow \text { inv })}{\Gamma_{S M}+\Gamma(h \rightarrow \text { inv })}$, as functions of the scalar mass and with three values for $\bar{\lambda}^{\prime}=5 \times 10^{-4}$, $1 \times 10^{-3}$ and $9 \times 10^{-3}$ for the mass range $m_{h_{2}^{0}}<62 \mathrm{GeV}$. Note that the curve for $\bar{\lambda}^{\prime}=0.009$ is excluded if we want to have a dark matter candidate and the invisible branching ratio $\mathscr{B}(h \rightarrow D M)<0.2$ [38].

Since $\bar{\lambda}^{\prime}=\lambda_{5}+\lambda_{6}+2 \lambda_{7}$, from the constraints in Eq. (29) and from the expressions for the masses in Eq. (8) and Eq. (11), we have

$$
\frac{2\left(m_{H}^{2}-\mu_{d}^{2}\right)}{v_{S M}^{2} \sqrt{\lambda_{4}\left(\lambda_{1}+\lambda_{3}\right)}}>-1, \quad \frac{\left(4 m_{c}^{2}-2 \mu_{d}^{2}\right)}{v_{S M}^{2} \sqrt{\lambda_{4}\left(\lambda_{1}+\lambda_{3}\right)}}>-1 .
$$




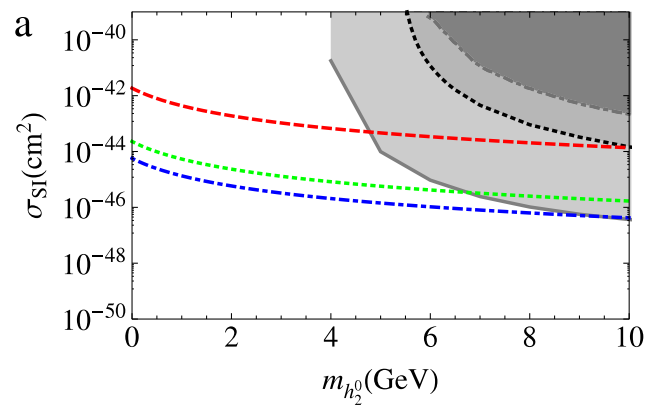

$\ldots \quad \lambda^{\prime}=0.001$

--- $\lambda^{\prime}=0.009$

- LUX

XENON100

- XENON1T

-.- $\lambda^{\prime}=0.0005$

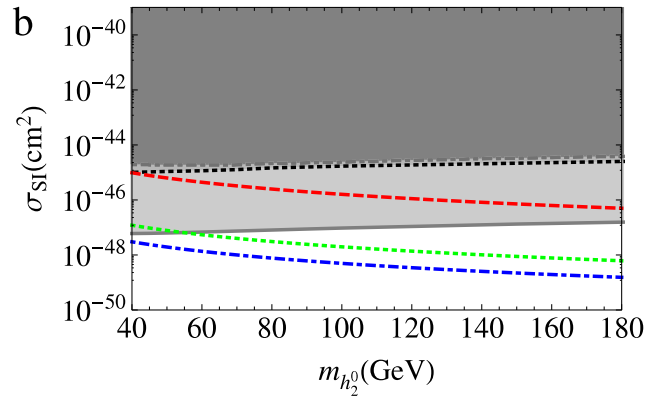

$=-. \quad \lambda^{\prime}=0.001$

--- $\lambda^{\prime}=0.009$

- LUX

- XENON100

- XENON1T

-. $\lambda^{\prime}=0.0005$

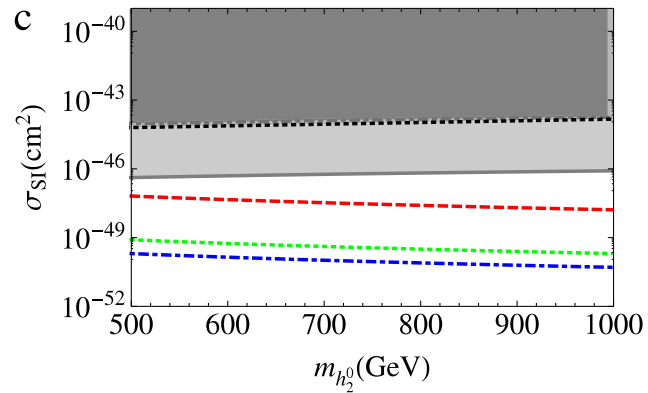

-.-- $\lambda^{\prime}=0.001$

-- $\lambda^{\prime}=0.009$

- LUX

- XENON100

- XENON1T

-.. $\lambda^{\prime}=0.0005$

Fig. 1. The gray areas show the regions excluded by Xenon, Lux and the theoretical prediction Xenon1T for $\sigma_{S I}$ defined in Eq. (31) as a function of the DM-candidate mass, and for three different values of $\bar{\lambda}^{\prime}$. (a) is the region for masses less than 10 $\mathrm{GeV}$, (b) shows the behavior of Eq. (31) for masses between 40 and $160 \mathrm{GeV}$, finally in (c) we show the allowed region for masses larger than $500 \mathrm{GeV}$.

From Eq. (33), we obtain the allowed region for $\mu_{d}^{2}$ and $\left(\lambda_{1}+\lambda_{3}\right)$ if we fix $m_{H}^{2}$ and $m_{c}^{2}$, and $\lambda_{4}=0.13$ is fixed by the SM Higgs mass. These are shown in Fig. 3 for (a) $m_{H}^{2}=54.1$ and $m_{c}^{2}=85$, (b) $m_{H}^{2}=80$ and $m_{c}^{2}=95$, and (c) $m_{H}^{2}=168$ and $m_{c}^{2}=84.7$. These values are also compatible with the experimental data of dark matter, as was shown in Ref. [43].

The presence of two inert doublet implies in contributions for $h^{0} \rightarrow \gamma \gamma$ [44], and $h^{0} \rightarrow Z \gamma$ [45]. In the latter paper it was obtained the best value for $\lambda_{5}$, that fit the current data for $h \rightarrow \gamma \gamma$, when it is $\lambda_{5}=-0.4$, in Fig. 4 we show the constraints on $\lambda_{1}$ and $\lambda_{3}$ using the third line of Eq. (29), $\left(\lambda_{5}+\sqrt{\lambda_{4}\left(\lambda_{1}+\lambda_{3}\right)}>0\right)$.

\section{Conclusions}

If the Higgs sector has, as in the fermion sector, three sequential generations, we should expect the existence of extra symmetries to make the interactions and the mass spectra simplest in the scalar sector. This is because, in general, three Higgs doublet models have complicated scalar potentials and 

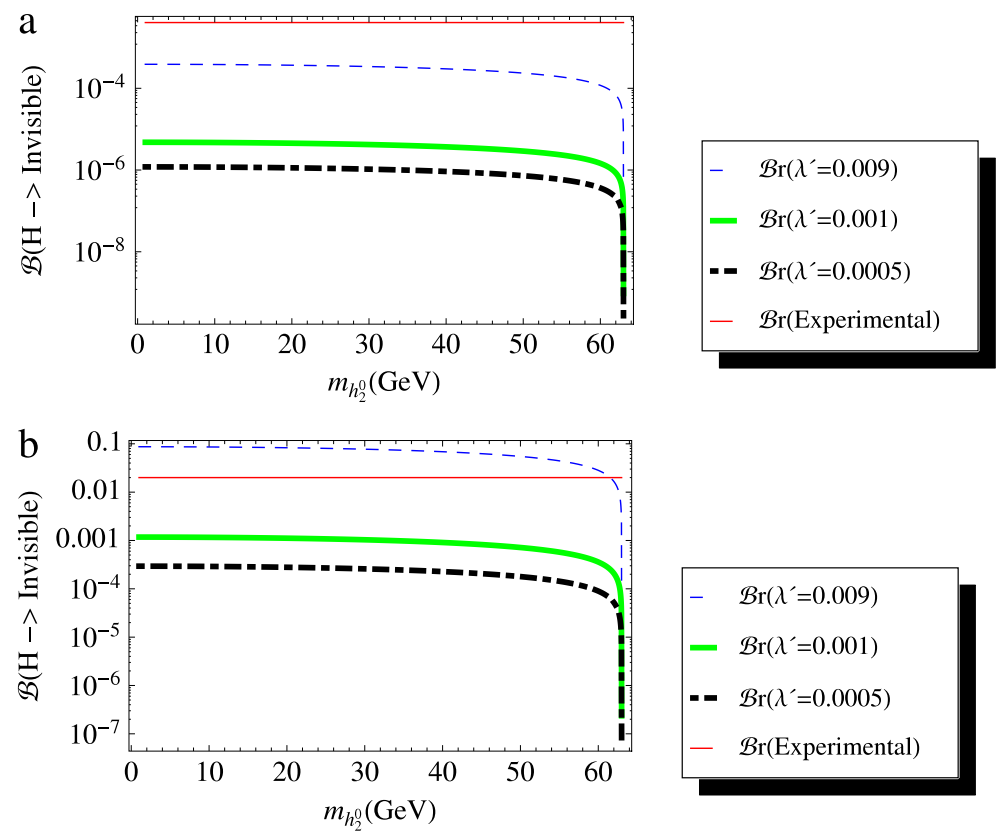

Fig. 2. The invisible Higgs width (a) defined in Eq. (32), and the branching ratio (b), $B r\left(h \rightarrow\right.$ inv) $=\frac{\Gamma(h \rightarrow \text { inv) }}{\Gamma_{S M}+\Gamma(h \rightarrow \text { inv) }}$ as functions of the scalar mass with $\bar{\lambda}^{\prime}=5 \times 10^{-4}, \bar{\lambda}^{\prime}=9 \times 10^{-3}$ and $\bar{\lambda}^{\prime}=10^{-3}$ for masses in the range $m_{h}<62 \mathrm{GeV}$. As can be seen $\bar{\lambda}^{\prime}=9 \times 10^{-3}$ is excluded by data, if we want that the scalar be a dark matter candidate.

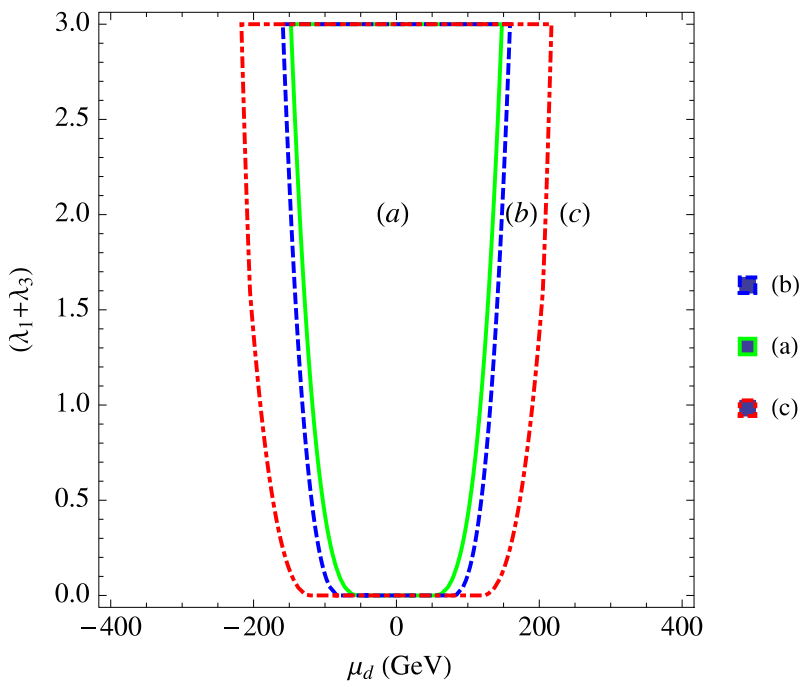

Fig. 3. The allowed region, using Eq. (33), for $\mu_{d}^{2}$ and $\left(\lambda_{1}+\lambda_{3}\right)$ when we fix (a) $m_{H}^{2}=54.1$ and $m_{c}^{2}=85$, (b) $m_{H}^{2}=80$ and $m_{c}^{2}=95$, and (c) $m_{H}^{2}=168$ and $m_{c}^{2}=84.7$. With $\lambda_{4}=0.13$ fixed by the SM Higgs mass.

mass matrices in each charge sector are diagonalized by arbitrary unitary $3 \times 3$ matrices having each one three mixing angles and six phases (some of them may be absorbed). In the present model because of the $S_{3}$ symmetry and the vacuum alignment, the entries of the rotation matrices are, at the tree level, Clebsch-Gordan-like coefficients. This eliminate plenty of new parameters that should have to 


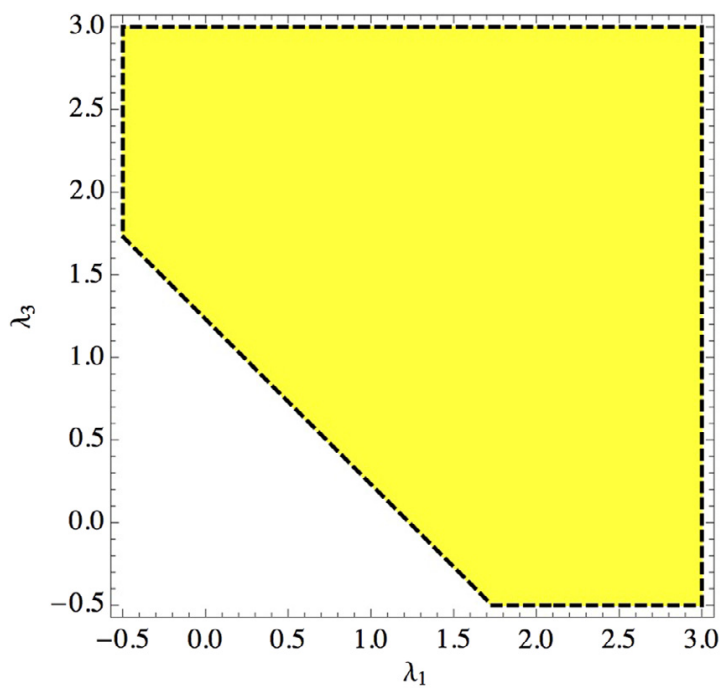

Fig. 4. Using Eq. (29), $\lambda_{5}>-\sqrt{\lambda_{4}\left(\lambda_{1}+\lambda_{3}\right)}$, and the results in Fig. 3 we obtain the allowed region for $\lambda_{1}$ and $\lambda_{3}$ when we fix $\lambda_{5}=-0.4$ and $\lambda_{4}=0.13$ is fixed by the SM Higgs mass, is the yellow area in figure above.

be determined by experiments. In fact, the scalar potential in this models is as simple as that in a general two Higgs doublet model. The only difference is the $\lambda_{8}$ term in the scalar potential, see Eq. (1) or (2). Anyway it is necessary that $\lambda_{8}=0$ in order to maintain the inert character of the doublet of $S_{3}, D$.

Moreover, like multi-Higgs models with no flavor changing neutral currents mediated by neutral scalars, the only mixing parameters appearing in the fermion charged interactions are the CKM and PMNS angles and phases. For more details see Ref. [44]. We would like to stress that the existence of two inert doublets, and the flavor conservation in the neutral currents mediated by scalars are consequences of three ingredients: (i) the $S_{3}$ symmetry, (ii) the representation content of the fermion and scalar multiplets under $S_{3}$, and, (iii) the vacuum alignment.

If the lightest neutral scalars are the $C P$-even as we have assumed here, the $C P$-odd ones can be produced at LHC in vector-boson fusion. This also deserves a detailed study.

\section{Acknowledgments}

The authors would like to thank CAPES under the PNPD Program (ACBM) and CNPq for full support under the contract 305991/2011-6.

\section{Appendix A. Constraint equations in model A}

Expanding the scalar potential in Eq. (1) as a function of VEV's, we will obtain:

$$
\begin{aligned}
V= & \frac{1}{36}\left(6 \mu_{s}^{2}\left(v_{1}+v_{2}+v_{3}\right)^{2}+12 \mu_{d}^{2}\left(v_{1}^{2}+v_{2}^{2}-v_{2} v_{3}+v_{3}^{2}-v_{1}\left(v_{2}+v_{3}\right)\right)\right. \\
& +4 \lambda_{1}\left(v_{1}^{2}+v_{2}^{2}-v_{2} v_{3}+v_{3}^{2}-v_{1}\left(v_{2}+v_{3}\right)\right)^{2}+4 \lambda_{3}\left(v_{1}^{2}+v_{2}^{2}-v_{2} v_{3}+v_{3}^{2}\right. \\
& \left.\left.-v_{1}\left(v_{2}+v_{3}\right)\right)^{2}\right)+\lambda_{4}\left(v_{1}+v_{2}+v_{3}\right)^{4}+2 \lambda_{5}\left(v_{1}+v_{2}+v_{3}\right)^{2}\left(v_{1}^{2}+v_{2}^{2}-v_{2} v_{3}+v_{3}^{2}\right. \\
& \left.-v_{1}\left(v_{2}+v_{3}\right)\right)+2 \lambda_{6}\left(v_{1}+v_{2}+v_{3}\right)^{2}\left(v_{1}^{2}+v_{2}^{2}-v_{2} v_{3}+v_{3}^{2}-v_{1}\left(v_{2}+v_{3}\right)\right) \\
& +4 \lambda_{7}\left(v_{1}+v_{2}+v_{3}\right)^{2}\left(v_{1}^{2}+v_{2}^{2}-v_{2} v_{3}+v_{3}^{2}-v_{1}\left(v_{2}+v_{3}\right)\right) \\
& -2 \sqrt{2} \lambda_{8}\left(v_{1}+v_{2}-2 v_{3}\right)\left(2 v_{1}-v_{2}-v_{3}\right)\left(v_{1}-2 v_{2}+v_{3}\right)\left(v_{1}+v_{2}+v_{3}\right),
\end{aligned}
$$


the constraint equations are explicitly given by:

$$
\begin{aligned}
18 t_{1}= & 6 \mu_{d}^{2}\left(2 v_{1}-v_{2}-v_{3}\right)+6 \mu_{s}^{2} V+2\left(\Lambda_{1}-4 \sqrt{2} \lambda_{8}\right) v_{1}^{3}-\left[\left(\Lambda_{2}+\sqrt{2} \lambda_{8}\right)\left(3 v_{1}^{2}+v_{2}^{2}+v_{3}^{2}\right)\right. \\
& \left.-\left(\Lambda_{3}-7 \sqrt{2} \lambda_{8}\right) v_{2} v_{3}\right]\left(v_{2}+v_{3}\right)+6\left[\left(\Lambda_{4}+2 \sqrt{2} \lambda_{8}\right)\left(v_{2}^{2}+v_{3}^{2}\right)\right. \\
& \left.+\left(\Lambda_{5}-2 \sqrt{2} \lambda_{8}\right) v_{2} v_{3}\right] v_{1} \\
18 t_{2}= & -\mu_{d}^{2}\left(v_{1}-2 v_{2}+v_{3}\right)+6 \mu_{s}^{2} V+2 \Lambda_{1} v_{2}^{3}+\left(\Lambda_{2}-\sqrt{2} \lambda_{8}\right)\left(v_{1}^{3}+v_{3}^{3}+3 v_{2}^{2} v_{3}+3 v_{2}^{2} v_{1}\right) \\
& +6\left(\Lambda_{4}+2 \sqrt{2} \lambda_{8}\right)\left(v_{1}^{2}+v_{3}^{2}\right) v_{2}+3\left(\Lambda_{5}-2 \sqrt{2} \lambda_{8}\right)\left(v_{1} v_{3}+2 v_{2} v_{3}+v_{3}^{2}\right) v_{1} \\
18 t_{3}= & -6 \mu_{d}^{2}\left(v_{1}+v_{2}-2 v_{3}\right)+\mu_{s}^{2} V+2 \Lambda_{1} v_{3}^{3}-\left(\Lambda_{2}+\sqrt{2} \lambda_{8}\right)\left(v_{1}^{3}+v_{2}^{3}+3 v_{1} v_{3}^{2}+3 v_{2} v_{3}^{2}\right) \\
& +6\left(\Lambda_{4}+2 \sqrt{2} \lambda_{8}\right)\left(v_{1}^{2}+v_{2}^{2}\right) v_{3}+3\left(\Lambda_{5}-2 \sqrt{2} \lambda_{8}\right)\left(v_{1} v_{2}+v_{2}^{2}+2 v_{2} v_{3}\right) v_{1}
\end{aligned}
$$

where

$$
\begin{aligned}
& \Lambda_{1}=2 \lambda^{\prime}+\lambda_{4}+2 \bar{\lambda}^{\prime}, \quad \Lambda_{2}=2 \lambda^{\prime}-2 \lambda_{4}-\bar{\lambda}^{\prime}, \quad \Lambda_{3}=2\left(\lambda^{\prime}+2 \lambda_{4}-2 \bar{\lambda}^{\prime}\right), \\
& \Lambda_{4}=\lambda^{\prime}+\lambda_{4}, \quad \Lambda_{5}=2 \lambda_{4}-\bar{\lambda}^{\prime} .
\end{aligned}
$$

Although the $\lambda_{8}$ term allows solutions, in this case the $\lambda_{8}$ symmetry has to be forbidden because it induces a tadpole that destabilize the vacuum alignment.

\section{Appendix B. Constraint equations in model B}

Expanding the scalar potential in Eq. (2) as a function of VEV's we have

$$
\begin{aligned}
V= & \frac{1}{4}\left(2 \mu_{s}^{2} v_{1}^{2}+\lambda_{4} v_{1}^{4}+v_{2}^{2}\left(2 \mu_{d}^{2}+\left(\lambda_{5}+\lambda_{6}+2 \lambda_{7}\right) v_{1}^{2}\right.\right. \\
& \left.-2 \lambda_{8} v_{1} v_{2}+\left(\lambda_{1}+\lambda_{3}\right) v_{2}^{2}\right)+\left(2 \mu_{d}^{2}+\left(\lambda_{5}+\lambda_{6}+2 \lambda_{7}\right) v_{1}^{2}\right. \\
& \left.\left.+6 \lambda_{8} v_{1} v_{2}+2\left(\lambda_{1}+\lambda_{3}\right) v_{2}^{2}\right) v_{3}^{2}+\left(\lambda_{1}+\lambda_{3}\right) v_{3}^{4}\right) .
\end{aligned}
$$

With the representation in Eq. (16), the constrain equation are

$$
\begin{aligned}
& 2 t_{1}=v_{1}\left[2 \mu_{s}^{2}+2 \lambda_{4} v_{1}^{2}+\bar{\lambda}^{\prime}\left(v_{2}^{2}+v_{3}^{2}\right)-\frac{\lambda_{8}}{v_{1}}\left(v_{2}^{3}+v_{2} v_{3}^{2}\right)\right], \\
& 2 t_{2}=v_{2}\left[2 \mu_{d}^{2}+\bar{\lambda}^{\prime} v_{1}^{2}+\left(\lambda_{1}+\lambda_{3}\right)\left(v_{2}^{2}+v_{3}^{2}\right)-3 \lambda_{8}\left(v_{1} v_{2}-\frac{v_{1} v_{3}^{2}}{v_{2}}\right)\right], \\
& 2 t_{3}=v_{3}\left[2 \mu_{d}^{2}+\bar{\lambda}^{\prime} v_{1}^{2}+2\left(\lambda_{1}+\lambda_{3}\right)\left(v_{2}^{2}+v_{3}^{2}\right)+6 \lambda_{8} v_{1} v_{2}\right],
\end{aligned}
$$

and we see that even in the general case when $v_{1} \neq v_{2} \neq v_{3}$ they are different from the respective equations in model A, see Eq. (A.2).

Notice that the $\lambda_{8}$ term avoids the zero solution for $v_{1}$ and $v_{2}$. If this term is forbidden with a $Z_{2}$ symmetry under which $D \rightarrow-D$ and all the other fields being even under this symmetry, we can have the solution $v_{1}=v_{S M}$ and $v_{2}=v_{3}=0$.

\section{References}

[1] G. Aad, et al., [ATLAS Collaboration], Phys. Lett. B 716 (2012) 1. arXiv:1207.7214 [hep-ex].

[2] S. Chatrchyan, et al., [CMS Collaboration], Phys. Lett. B 716 (2012) 30. arXiv:1207.7235 [hep-ex].

[3] T.D. Lee, Phys. Rev. D 8 (1973) 1226.

[4] Y.L. Wu, L. Wolfenstein, Phys. Rev. Lett. 73 (1994) 1762. [hep-ph/9409421].

[5] G.C. Branco, P.M. Ferreira, L. Lavoura, M.N. Rebelo, M. Sher, J.P. Silva, Phys. Rep. 516 (2012) 1. arXiv:1106.0034 [hep-ph], and references therein.

[6] S. Weinberg, Phys. Rev. Lett. 37 (1976) 657

[7] A.G. Dias, V. Pleitez, Phys. Rev. D 70 (2004) 055009. [hep-ph/0407074].

[8] B. Grzadkowski, O.M. Ogreid, P. Osland, A. Pukhov, M. Purmohammadi, J. High Energy Phys. 1106 (2011) 003. arXiv: 1012.4680 [hep-ph]. 
[9] J. Kubo, H. Okada, F. Sakamaki, Phys. Rev. D 70 (2004) 036007. [hep-ph/0402089].

[10] D. Das, U.K. Dey, Phys. Rev. D 89 (2014) 095025. arXiv:1404.2491 [hep-ph].

[11] S.-L. Chen, M. Frigerio, E. Ma, Phys. Rev. D 70 (2004) 073008; S.-L. Chen, M. Frigerio, E. Ma, Phys. Rev. D 70 (2004) 079905 (erratum) [hep-ph/0404084].

[12] N.G. Deshpande, E. Ma, Phys. Rev. D 18 (1978) 2574.

[13] R. Barbieri, L.J. Hall, V.S. Rychkov, Phys. Rev. D 74 (2006) 015007. arXiv:hep-ph/0603188.

[14] M. Cirelli, N. Fornengo, A. Strumia, Nuclear Phys. B 753 (2006) 178. arXiv:hep-ph/0512090.

[15] L. Lopez Honorez, E. Nezri, J.F. Oliver, M.H.G. Tytgat, J. Cosmol. Astropart. Phys. 0702 (2007) 028. arXiv:hep-ph/0612275.

[16] T. Hambye, M.H.G. Tytgat, Phys. Lett. B 659 (2008) 651. arXiv:0707.0633 [hep-ph].

[17] Q.H. Cao, E. Ma, G. Rajasekaran, Phys. Rev. D 76 (2007) 095011. arXiv:0708.2939 [hep-ph].

[18] S. Andreas, T. Hambye, M.H.G. Tytgat, J. Cosmol. Astropart. Phys. 0810 (2008) 034. arXiv:0808.0255 [hep-ph].

[19] E. Lundstrom, M. Gustafsson, J. Edsjo, Phys. Rev. D 79 (2009) 035013. arXiv:0810.3924 [hep-ph].

[20] T. Hambye, F.S. Ling, L. Lopez Honorez, J. Rocher, J. High Energy Phys. 0907 (2009) 090. arXiv:0903.4010 [hep-ph].

[21] L. Lopez-Honorez, C.E. Yaguna, J. Cosmol. Astropart. Phys. 1101 (2011) 002. arXiv:1011.1411 [hep-ph].

[22] M. Krawczyk, D. Sokolowska, P. Swaczyna, B. Swiezewska, J. High Energy Phys. 1309 (2013) 055. arXiv:1305.6266 [hep-ph].

[23] Y. Daikoku, H. Okada, T. Toma, Progr. Theoret. Phys. 126 (2011) 855. arXiv:1106.4717 [hep-ph].

[24] A. Biswas, D. Majumdar, A. Sil, P. Bhattacharjee, J. Cosmol. Astropart. Phys. 1312 (2013) 049. arXiv:1301.3668 [hep-ph].

[25] S. Bhattacharya, A. Drozd, B. Grzadkowski, J. Wudka, J. High Energy Phys. 1310 (2013) 158. arXiv:1309.2986 [hep-ph].

[26] For the representations and rules of the $S_{3}$ symmetry see H. Ishimori, T. Kobayashi, H. Ohki, Y. Shimizu, H. Okada, M. Tanimoto, Progr. Theoret. Phys. Suppl. 183 (2010) 1. arXiv:1003.3552 [hep-th].

[27] A. Mondragon, M. Mondragon, E. Peinado, Phys. Rev. D 76 (2007) 076003. arXiv:0706.0354 [hep-ph].

[28] O.F. Beltran, M. Mondragon, E. Rodriguez-Jauregui, J. Phys. Conf. Ser. 171 (2009) 012028.

[29] J.F. Gunion, H.E. Haber, Phys. Rev. D 67 (2003) 075019. [hep-ph/0207010].

[30] A. Goudelis, B. Hermanniea, O. Stal, J. High Energy Phys. 1309 (2013) 106. arXiv:1303.3010 [hep-ph].

[31] G. Altarelli, PoS HRMS 2010 (2010) 022. arXiv:1011.5342 [hep-ph].

[32] S. Pakvasa, H. Sugawara, Phys. Lett. B 73 (1978) 61.

[33] G. Bhattacharyya, P. Leser, H. Pas, Phys. Rev. D 83 (2011) 011701. arXiv:1006.5597 [hep-ph].

[34] T. Teshima, Phys. Rev. D 85 (2012) 105013. arXiv:1202.4528 [hep-ph].

[35] S. Kaneko, H. Sawanaka, T. Shingai, M. Tanimoto, K. Yoshioka, Progr. Theoret. Phys. 117 (2007) 161. [hep-ph/0609220].

[36] S.L. Glashow, S. Weinberg, Phys. Rev. D 15 (1977) 1958.

[37] K. Kannike, Eur. Phys. J. C 72 (2012) 2093. arXiv: 1205.3781 [hep-ph].

[38] G. Belanger, B. Dumont, U. Ellwanger, J.F. Gunion, S. Kraml, Phys. Lett. B 723 (2013) 340. arXiv:1302.5694 [hep-ph].

[39] Y. Mambrini, Phys. Rev. D 84 (2011) 115017. arXiv:1108.0671 [hep-ph].

[40] S. Chatrchyan, et al., [CMS Collaboration], Eur. Phys. J. C 74 (2014) 2980. arXiv:1404.1344 [hep-ex].

[41] E. Aprile, et al., [XENON100 Collaboration], Phys. Rev. Lett. 109 (2012) 181301. arXiv:1207.5988 [astro-ph.CO].

[42] D.S. Akerib, et al., [LUX Collaboration], Phys. Rev. Lett. 112 (2014) 091303. arXiv:1310.8214 [astro-ph.CO].

[43] E.C.F.S. Fortes, A.C.B. Machado, J. Montaño, V. Pleitez, 2014. Scalar Dark Matter Candidates in Two Inert Higgs Doublet Model, arXiv: 1407.4749 [hep-ph].

[44] H. Cardenas, A.C.B. Machado, V. Pleitez, J. Alexis Rodriguez, Phys. Rev. D 87 (2013) 035028. arXiv:1212.1665 [hep-ph].

[45] E.C.F.S. Fortes, A.C.B. Machado, J. Montaño, V. Pleitez, 2014. Two inert scalar doublet model and $h \rightarrow \gamma \gamma, \gamma Z$ at LHC, arXiv: 1408.0780 [hep-ph]. 